\title{
Radiation reaction effects on the interaction of an electron with an intense laser pulse
}

\author{
Yevgen Kravets, Adam Noble, ${ }^{*}$ and Dino Jaroszynski \\ Department of Physics, SUPA and University of Strathclyde, Glasgow, G4 ONG, United Kingdom
}

(Received 3 April 2013; published 30 July 2013)

\begin{abstract}
Radiation reaction effects will play an important role in near-future laser facilities, yet their theoretical description remains obscure. We explore the Ford-O'Connell equation for radiation reaction, and discuss its relation to other commonly used treatments. By analyzing the interaction of a high energy electron in an intense laser pulse, we find that radiation reaction effects prevent the particle from accessing a regime in which the Landau-Lifshitz approximation breaks down.
\end{abstract}

DOI: 10.1103/PhysRevE.88.011201

PACS number(s): 41.60.-m, 41.75.Jv

\section{INTRODUCTION}

The question of how an accelerating charged particle interacts with the radiation it produces remains unclear, despite investigations stretching back more than a century. Until recently, this issue has been motivated principally by theoretical curiosity, since the radiation reaction force is in general a negligible correction to the Lorentz force from the external fields. However, with the advent of modern ultraintense laser facilities, solving this problem is becoming a real practical concern. At the Extreme Light Infrastructure (ELI), for instance, it is envisaged that laser pulses will be produced with intensities exceeding $10^{23} \mathrm{~W} / \mathrm{cm}^{2}$, and interact with $\mathrm{GeV}$ or higher energy electrons [1]. In such regimes, the radiation reaction force may be comparable to and even exceed the applied force.

The problem of radiation reaction was first addressed by Lorentz [2] and Abraham [3], on the basis of a rigid charged sphere. It was later made fully relativistic by Dirac [4], who treated the electron as a point particle, and started from the principles of energy-momentum conservation. The resulting equation, now known as the Lorentz-Abraham-Dirac (LAD) equation, reads

$$
\ddot{x}^{a}=-\frac{q}{m} F_{b}^{a} \dot{x}^{b}+\tau \Delta^{a}{ }_{b} \dddot{x}^{b} .
$$

Here, $q$ and $m$ are the charge and mass and $\tau:=q^{2} / 6 \pi m \simeq$ $6 \times 10^{-24} \mathrm{~s}$ is the characteristic time of the electron (in Heaviside-Lorentz units with $c=1) . F_{a b}$ are components of the external electromagnetic field, $\Delta^{a}{ }_{b}:=\delta_{b}^{a}+\dot{x}^{a} \dot{x}_{b}$ is the $\dot{x}$-orthogonal projection, and an overdot denotes differentiation with respect to proper time $s$. We use the Einstein summation convention and raise and lower indices with the metric tensor $\eta_{a b}=\operatorname{diag}(-1,1,1,1)$.

There are many conceptually distinct derivations [4-12] of the LAD equation (1). However, it is plagued with difficulties. Generic initial conditions lead to exponentially growing accelerations ("runaway solutions") even in the absence of external forces, and these can only be eliminated by choosing initial conditions that depend on the motion of the charge at all subsequent times, violating causality.

The usual approach to overcoming the problems of the LAD equation is to treat the radiation reaction as a small perturbation

*adam.noble@strath.ac.uk to the applied Lorentz force, and keep only terms up to order $\tau$. Thus the problematic third derivative ("jerk") term in (1) can be replaced by the derivative of the Lorentz force, yielding

$$
\ddot{x}^{a}=-\frac{q}{m} F_{b}^{a} \dot{x}^{b}-\tau \frac{q}{m} \dot{x}^{c} \partial_{c} F_{b}^{a} \dot{x}^{b}+\tau \frac{q^{2}}{m^{2}} \Delta_{b}^{a} F_{c}^{b} F_{d}^{c} \dot{x}^{d} .
$$

This equation, first introduced in [13] and known as the Landau-Lifshitz (LL) equation, is free from the difficulties of runaway solutions and preacceleration that beset (1). Additional support for the LL equation can be found [14,15], but always in the perturbative regime, and there has recently been a renewed interest in assessing the validity of the LL equation [16-18].

A number of other descriptions of radiating electrons have been proposed [19-21], and received varying degrees of interest. One such description [22,23], introduced by Ford and $\mathrm{O}^{\prime} \mathrm{Connell}$, is derived by abandoning the assumption that the electron is a point particle, and choosing instead a minimal form factor consistent with causality.

In the presence of an arbitrary external force $f^{a}$, the FordO'Connell (FO) equation reads

$$
\ddot{x}^{a}=f^{a}+\tau \Delta^{a}{ }_{b} \dot{f}^{b} .
$$

The appearance of the projection $\Delta^{a}{ }_{b}$ in (3) ensures that the normalization of the 4-velocity,

$$
\dot{x} \cdot \dot{x}=-1,
$$

is preserved.

The FO equation has received less attention than it perhaps deserves, due in part to confusion arising from its apparent equivalence to the LL approximation [24]. However, because the FO equation is regarded as exact, we cannot replace any acceleration terms arising from the derivative of the applied force with the applied force itself, as is done in the LL prescription.

One might question the value of the FO equation: if the electron has the specific structure used in its derivation then it is the exact equation (in the classical limit) for the motion of an electron; otherwise it is just one further approximate description. However, since it is an intermediate step in the derivation of (2) from (1), any discrepancy between the predictions of FO and LL implies a failure of the latter. A direct comparison between predictions of LL and LAD is in general not possible, due to the occurrence of runaways.

In this Rapid Communication, we explore the detailed form of the FO equation in an ambient electromagnetic field. We 
then compare its predictions with those of the LL equation for the case of a particle interacting with a laser pulse, modeling the latter as a plane wave, and find good agreement even where one might a priori expect the LL description to break down. This results from rapid radiative losses while the particle is still in a low field region, and suggests there may be limitations on the energies of a particle within an ultraintense laser pulse. This could have implications for attempts to probe the quantum vacuum [25].

\section{FORD-O'CONNELL EQUATION}

The dominant forces on a classical charged particle are electromagnetic, so we use the Lorentz force $f^{a}=-\frac{q}{m} F^{a}{ }_{b} \dot{x}^{b}$ as the applied force in (3), whence the Ford-O'Connell equation takes the form

$$
\left(\Delta_{b}^{a}+\tau G_{b}^{a}\right) \ddot{x}^{b}=-\frac{q}{m}\left(F_{b}^{a}+\tau \dot{x}^{c} \partial_{c} F_{b}^{a}\right) \dot{x}^{b},
$$

where $G^{a}{ }_{b}=\frac{q}{m} \Delta^{a}{ }_{c} F^{c}{ }_{d} \Delta^{d}{ }_{b}$. Apart from the term involving derivatives of the fields, this coincides with the Mo-Papas equation [19]. The latter was derived heuristically, rather than either from first principles or as an approximation to the LAD equation.

In order for (5) to be a valid equation of motion, it is necessary that it can be solved for the acceleration $\ddot{x}$. Writing it as

$$
M_{b}^{a} \ddot{x}^{b}=-\frac{q}{m}\left(F_{b}^{a}+\tau \dot{x}^{c} \partial_{c} F_{b}^{a}\right) \dot{x}^{b},
$$

we therefore need to show that $M$ can be inverted. However, care must be taken in defining the inverse: taken as a matrix acting on all 4 -vectors, $M$ annihilates $\dot{x}$, and therefore cannot be inverted. However, from (5), we only require $M$ to act on (and produce) vectors orthogonal to $\dot{x}$, hence we can define its inverse by

$$
\left(M^{-1}\right)_{b}^{a} M_{c}^{b}=M_{b}^{a}\left(M^{-1}\right)^{b}{ }_{c}=\Delta^{a}{ }_{c},
$$

and its determinant by

$$
\operatorname{det} M=\frac{1}{3 !} \varepsilon_{a b c d} \varepsilon^{e f g h} M^{a}{ }_{e} M^{b}{ }_{f} M^{c}{ }_{g} \dot{x}^{d} \dot{x}_{h} .
$$

From this it follows that

$$
\operatorname{det} M=1+\frac{\tau^{2}}{2} G^{a b} G_{a b}
$$

In terms of the fields "seen" by the particle, this is det $M=$ $1+\tau^{2} \frac{q^{2}}{m^{2}} B^{2}>0$, and the FO equation is a viable description for the motion of a charged particle.

It can readily be seen that, ignoring terms of order $\tau^{2}$ or higher, the FO and LL equations coincide. For the LL equation to be a good approximation to FO, then, it is necessary that

$$
\tau \sqrt{G^{a b} G_{a b} / 2} \ll 1,
$$

though this involves only the magnetic field seen by the particle, which does not contribute to the applied force. The condition (10) is necessary, but not sufficient. However, we focus on this scalar condition, as it is more readily applicable than the somewhat vague requirement that $M_{b}^{a}$ is "close" to the unit matrix.

\section{PARTICLE MOTION IN A PLANE WAVE}

Radiation reaction effects will be most prominent for high energy electrons interacting with ultraintense laser pulses [26,27]. Although realistic laser pulses have important transverse structure, for electrons co- or counterpropagating approximately through the center of the pulse these are largely unimportant, so for simplicity we will consider a plane wave of the form

$$
\frac{q}{m} F_{a b}=\mathcal{E}\left(\epsilon_{a} n_{b}-\epsilon_{b} n_{a}\right),
$$

where $\epsilon$ is the polarization, $n_{a}=(1,-\mathbf{n})$ is the (null) propagation direction, and the electric field $\frac{m}{q} \mathcal{E}$ depends only on $n_{a} x^{a}$. The polarization and propagation directions satisfy

$$
\epsilon \cdot \epsilon=1, \quad \epsilon \cdot n=0, \quad n \cdot n=0 .
$$

For definiteness we have assumed linear polarization, though the analysis is readily generalized.

In the absence of radiation reaction, the solution to the Lorentz force equation $\ddot{x}^{a}=-\frac{q}{m} F^{a}{ }_{b} \dot{x}^{b}$ in a plane electromagnetic wave (11) is well known: particularly lucid accounts may be found in $[28,29]$. Analytical solutions also exist for the LL equation [26,30,31].

Assuming the electron's motion is in the spatial plane spanned by $\epsilon$ and $n$, and defining the coordinates

$$
\phi=n_{a} x^{a}, \quad \xi=\epsilon_{a} x^{a}, \quad \psi=m_{a} x^{a},
$$

where $m_{a}=(1, \mathbf{n})$, the FO equation yields, after some manipulation,

$$
\begin{gathered}
\ddot{\phi}=-\tau \frac{\mathcal{E}+\tau \dot{\phi} \mathcal{E}^{\prime}}{1+\tau^{2} \mathcal{E}^{2} \dot{\phi}^{2}} \mathcal{E} \dot{\phi}^{3}, \\
\ddot{\xi}=-\dot{\phi} \frac{\mathcal{E}+\tau \dot{\phi} \mathcal{E}^{\prime}}{1+\tau^{2} \mathcal{E}^{2} \dot{\phi}^{2}}(1+\tau \mathcal{E} \dot{\xi} \dot{\phi}),
\end{gathered}
$$

where a prime denotes differentiation with respect to $\phi$. The other dynamical component of $x, \psi$, can be obtained from the normalization condition (4), which takes the form

$$
1=\dot{\phi} \dot{\psi}-\dot{\xi}^{2}
$$

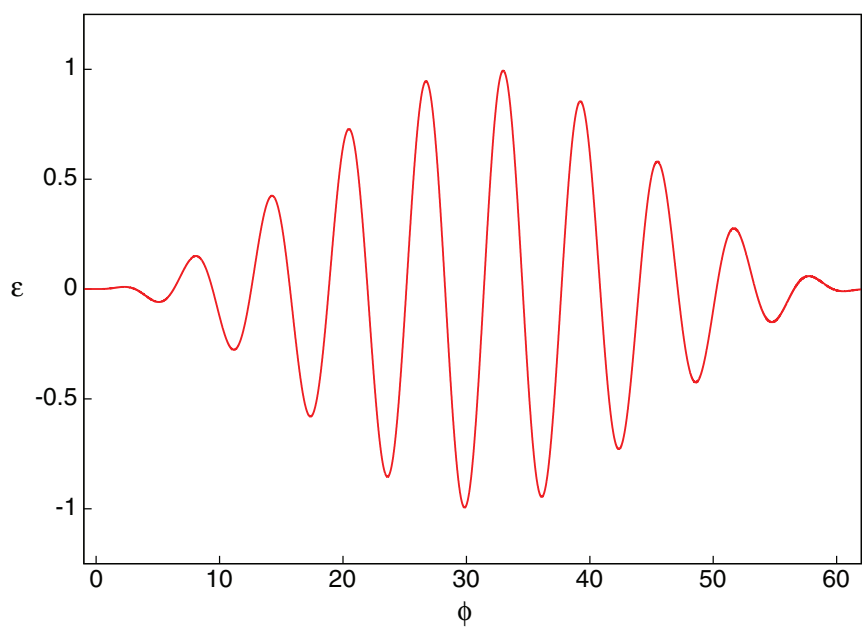

FIG. 1. (Color online) Electric field $\mathcal{E}$ [Eq. (19)] as a function of $\phi$, for $N=10, a_{0}=1$, in units such that $\omega=1$. 
It follows that the electron's energy, normalized to $m c^{2}$, is given by

$$
\gamma=\frac{1}{2}(\dot{\phi}+\dot{\psi})=\frac{1+\dot{\phi}^{2}+\dot{\xi}^{2}}{2 \dot{\phi}} .
$$

To first order in $\tau,(14)$ and (15) reduce to their counterparts in the LL equation, as expected, and are consistent with QED [32]. We are interested in exploring conditions under which this approximation breaks down, and whether this can be realized in the foreseeable future.

From (10), it follows that the LL equation should be reliable only when $\tau \sqrt{G^{a b} G_{a b} / 2} \ll 1$, or in the plane wave (11),

$$
\mathcal{T}:=\tau \mathcal{E} \dot{\phi} \ll 1,
$$

as is clearly borne out by Eqs. (14) and (15).

To compare the predictions of FO and LL in a plane wave, we need to specify the profile of the electric field $\mathcal{E}$, though the specific choice does not significantly affect the results. It will be convenient to choose $\mathcal{E}$ to have compact support, so the electron can begin and end in vacuum. Furthermore, both $\mathcal{E}$ and its derivative should be continuous. We adopt the simple
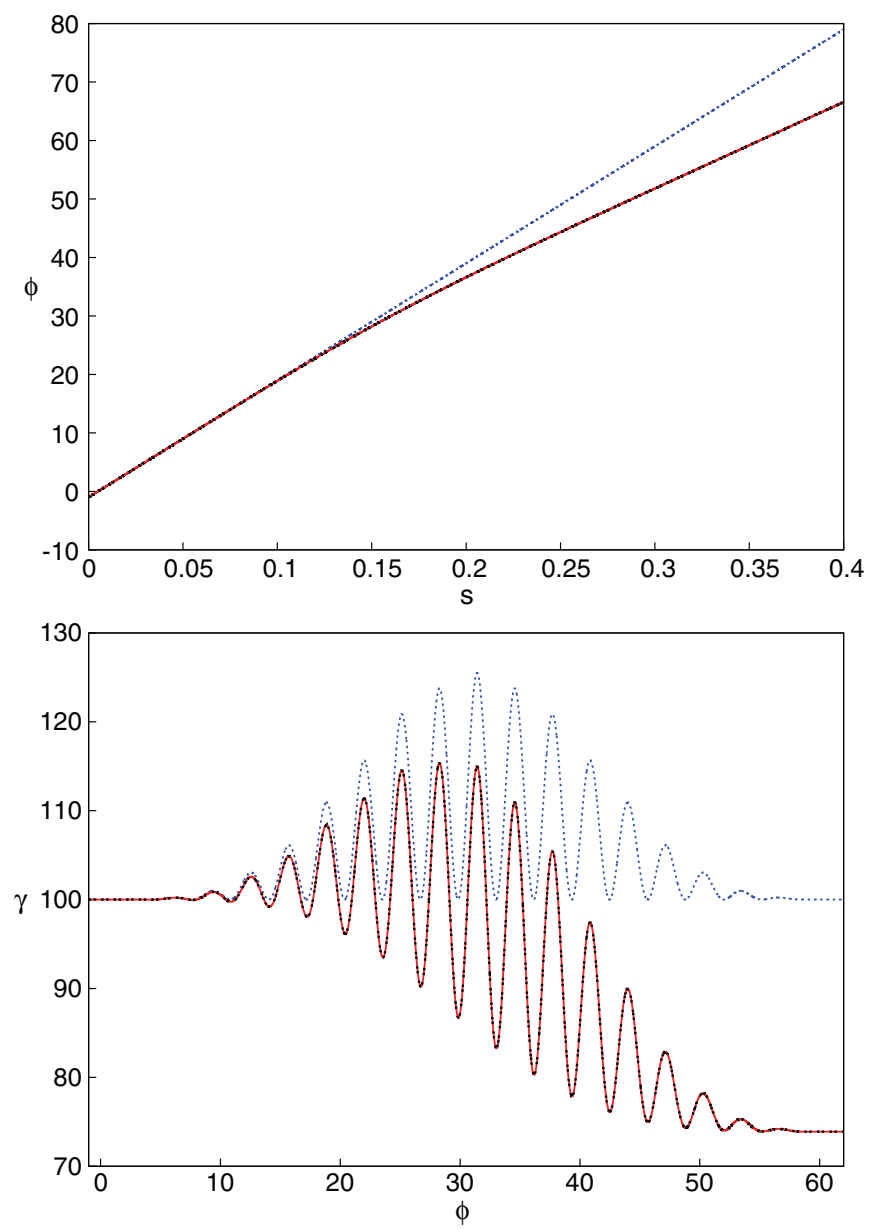

FIG. 2. (Color online) Radiation reaction effects on an electron of initial energy $\gamma_{\text {in }}=100$ colliding head-on with a pulse with $a_{0}=100$. Upper panel: $\phi$ as a function of $s$. Lower panel: $\gamma$ as a function of $\phi$. Dotted blue curves, without radiation reaction; solid red curves, with LL radiation reaction; double-dotted black curves, with FO radiation reaction. choice (related to profiles used in, for example, [31,33,34]),

$$
\mathcal{E}= \begin{cases}\omega a_{0} \sin (\omega \phi) \sin ^{2}(\omega \phi / 2 N) & \text { for } 0<\phi<2 \pi N / \omega \\ 0 & \text { otherwise }\end{cases}
$$

This represents an $N$-cycle pulse of central frequency $\omega$, modulated by a $\sin ^{2}$-envelope. $a_{0}$ is the usual intensity parameter ("normalized vector potential"). Figure 1 shows (19) for $N=10$, in units such that $\omega=1$.

The function $\phi(s)$ is a useful measure of the rate at which the electron passes through the pulse, and thus, together with $\gamma$, is a good indication of the significance of radiation reaction. As Fig. 2 shows, for the highest currently attainable laser intensities $\left(a_{0}=100\right)$ and moderately high initial electron energies $(\gamma=100)$, radiation reaction has a significant effect, but LL and FO are in good agreement.

If we consider the most intense lasers under development $\left(a_{0} \sim 1000\right)$ and the highest energy electrons available $\left(\gamma \sim 10^{5}\right)$, we appear to be in a regime where the condition (18) is violated, and we would expect strong differences between LL and FO. However, as shown in Fig. 3, although the
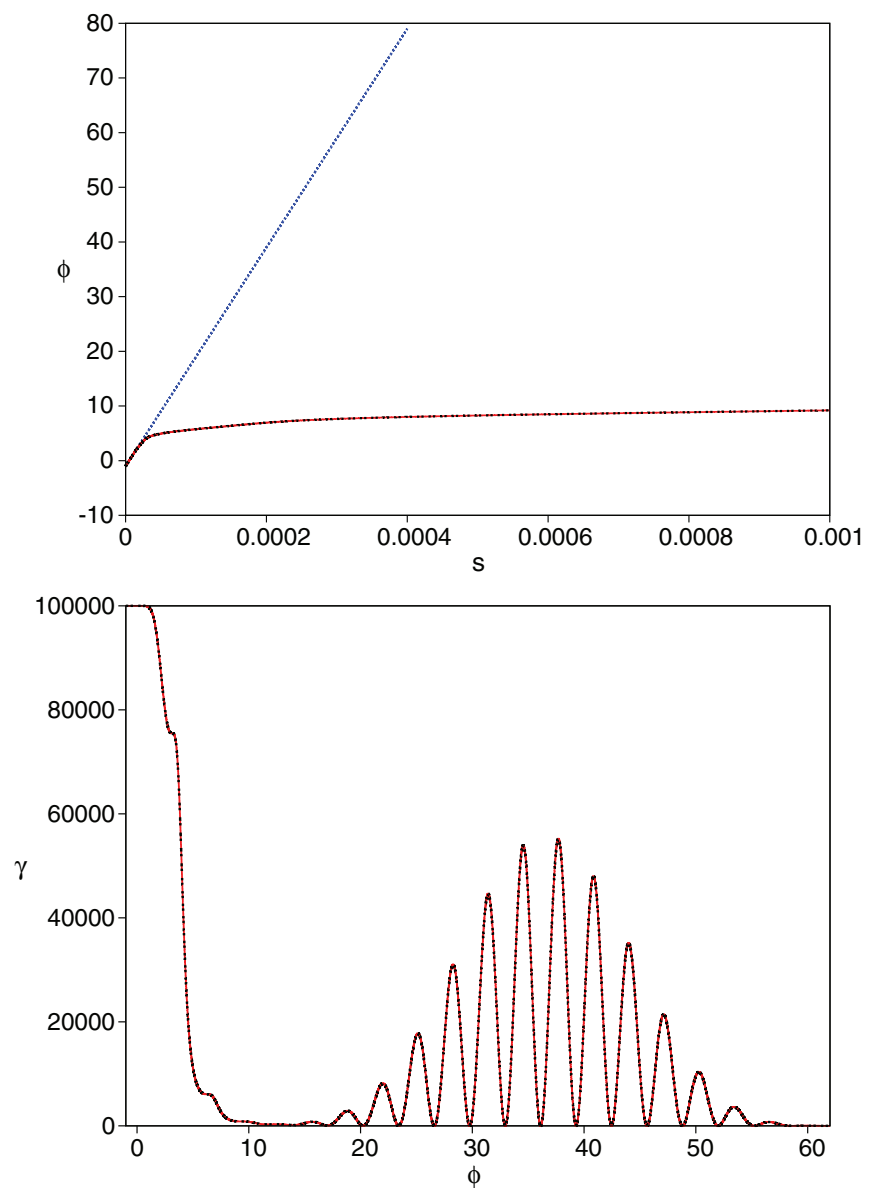

FIG. 3. (Color online) Radiation reaction effects on an electron of initial energy $\gamma_{\text {in }}=10^{5}$ colliding head-on with a pulse with $a_{0}=1000$. Upper panel: $\phi$ as a function of $s$. Lower panel: $\gamma$ as a function of $\phi$. Dotted blue curve, without radiation reaction; solid red curves, with LL radiation reaction; double-dotted black curves, with FO radiation reaction. 


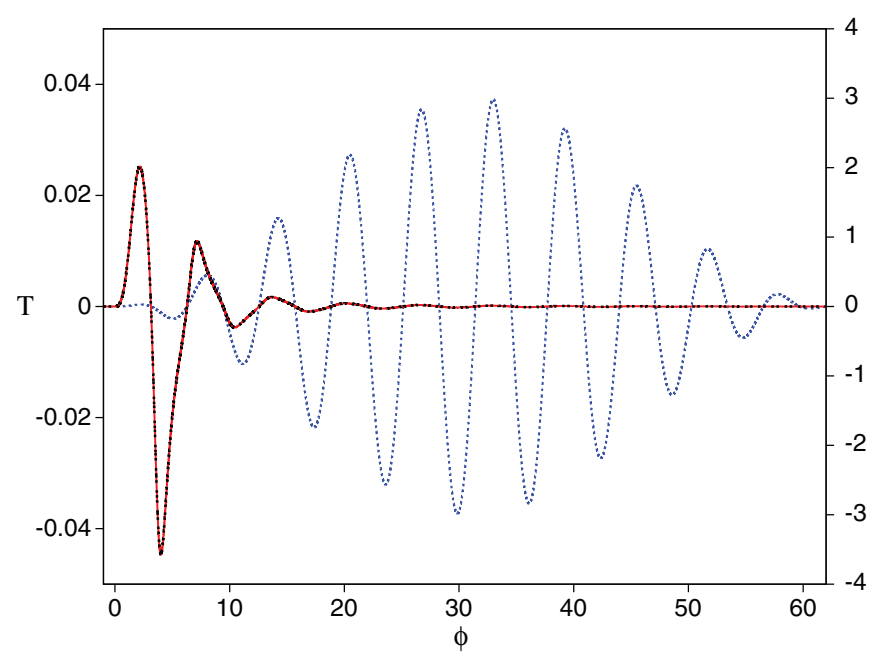

FIG. 4. (Color online) $\mathcal{T}$ as a function of $\phi$ with radiation reaction [left axis, solid red curve (LL), double-dotted black curve (FO)], and without radiation reaction (right axis, dotted blue curve).

dynamics is dominated by radiation reaction, agreement between the two theories remains strong. How are we to explain this?

The condition (18) refers to the instantaneous energy and field strength, whereas the previously quoted values of $a_{0}$ and $\gamma$ refer to the peak field and the initial energy. Since the particle begins in vacuum, initially $\mathcal{T}=0$. From Fig. 3 , it is clear that the electron loses almost all its energy to radiation in the first two cycles, while $\mathcal{E} \ll \omega a_{0}$. After this, it hardly radiates at all, and its evolution is well described by the Lorentz force alone, at a greatly reduced initial energy. As shown in Fig. 4, as the electron propagates through the laser pulse, its energy loss occurs at such a rate that $\mathcal{T}$ never approaches unity. Thus the Landau-Lifshitz equation remains a good description of radiation reaction phenomena for field strengths and electron energies far exceeding those currently proposed. Comparison with the values of $\mathcal{T}$ calculated for a (hypothetical) nonradiating particle demonstrates that the validity of the LL equation for such high energies is a direct consequence of radiation reaction (note the different scales in Fig. 4).

\section{CONCLUSION}

Radiation reaction will undoubtedly play an important role in upcoming laser facilities, such as ELI, and a proper understanding of its effects is essential for planning and interpreting results from experiments. The standard description of radiation reaction, the $\mathrm{LAD}$ equation, has major difficulties, and of the many alternatives suggested over the years, most are either ad hoc, or approximations whose validity needs to be established.

The equation of Ford and O'Connell has the advantage that it can be derived exactly, albeit for an electron of a specific given structure. It has the further advantage that, since it also arises as an intermediate step in the derivation of the LL equation from LAD, it can be used to test the validity of LL beyond the usual simple dimensional arguments. We have conducted a detailed exploration of the structure of the FO equation in electromagnetic fields, highlighting its relation to the LL and Mo-Papas treatments of radiation reaction.

By exploring the interaction of a high energy electron with an ultraintense laser pulse, we have shown that the radiation reaction effects as the electron enters the pulse ensure that it cannot enter a regime where the LL description ceases to be valid, even when a priori estimates would suggest otherwise. This implies a possible restriction on the energy of an electron penetrating a high intensity pulse, which may inhibit investigations of the QED vacuum.

It is worth noting that the analysis presented in this Rapid Communication assumes the laser pulse can be described by a plane wave. It is of interest to explore whether the results remain valid for a pulse with more realistic transverse structure. Also, we have neglected quantum effects $[35,36]$ and interactions with other electrons in a bunch [37]. These considerations will be addressed in future work.

\section{ACKNOWLEDGMENTS}

We are grateful to David Burton and Jonathan Gratus for many valuable discussions, and to Enrico Brunetti for technical assistance. We acknowledge support from SUPA, the Cockcroft Institute, the UK EPSRC, the EC's LASERLABEUROPE/LAPTECH (Grant Agreement No. 284464), and the Extreme Light Infrastructure (ELI) European Project.
[1] See http://www.extreme-light-infrastructure.eu/

[2] H. A. Lorentz, The Theory of Electrons and its Applications to the Phenomena of Light and Radiant Heat (Stechert, New York, 1916).

[3] M. Abraham, The Classical Theory of Electricity and Magnetism (Blackie, London, 1932).

[4] P. A. M. Dirac, Proc. R. Soc. London, Ser. A 167, 148 (1938).

[5] H. J. Bhabha, Proc. R. Soc. London, Ser. A 172, 384 (1939).

[6] J. A. Wheeler and R. P. Feynman, Rev. Mod. Phys. 17, 157 (1945).

[7] F. Rohrlich, Phys. Rev. Lett. 12, 375 (1964).

[8] C. Teitelboim, Phys. Rev. D 1, 1572 (1970).

[9] A. O. Barut, Phys. Rev. D 10, 3335 (1974).

[10] J. D. Jackson, Classical Electrodynamcis, 3rd ed. (Wiley, New York, 1999).
[11] D. A. Burton, J. Gratus, and R. W. Tucker, Ann. Phys. 322, 599 (2007).

[12] M. R. Ferris and J. Gratus, J. Math. Phys. 52, 092902 (2011).

[13] L. D. Landau and E. M. Lifshitz, The Classical Theory of Fields (Pergamon, London, 1962).

[14] H. Spohn, Europhys. Lett. 50, 287 (2000).

[15] S. E. Gralla, A. I. Harte, and R. M. Wald, Phys. Rev. D 80, 024031 (2009).

[16] D. J. Griffiths, T. C. Proctor, and D. F. Schroeter, Am. J. Phys. 78, 391 (2010).

[17] S. V. Bulanov, T. Zh. Esirkepov, M. Kando, J. K. Koga, and S. S. Bulanov, Phys. Rev. E 84, 056605 (2011).

[18] R. R. Pandit and Y. Sentoku, Phys. Plasmas 19, 073304 (2012).

[19] T. C. Mo and C. H. Papas, Phys. Rev. D 4, 3566 (1971). 
[20] I. V. Sokolov, N. M. Naumova, J. A. Nees, G. A. Mourou, and V. P. Yanovsky, Phys. Plasmas 16, 093115 (2009).

[21] R. T. Hammond, Phys. Rev. A 81, 062104 (2010).

[22] G. W. Ford and R. F. O'Connell, Phys. Lett. A 157, 217 (1991).

[23] G. W. Ford and R. F. O'Connell, Phys. Lett. A 174, 182 (1993).

[24] R. F. O’Connell, Phys. Lett. A 313, 491 (2003).

[25] A. Di Piazza, C. Müller, K. Z. Hatsagortsyan, and C. H. Keitel, Rev. Mod. Phys. 84, 1177 (2012).

[26] Y. Hadad, L. Labun, J. Rafelski, N. Elkina, C. Klier, and H. Ruhl, Phys. Rev. D 82, 096012 (2010).

[27] G. Lehmann and K. H. Spatschek, Phys. Rev. E 84, 046409 (2011).

[28] J. W. Meyer, Phys. Rev. D 3, 621 (1971).

[29] T. Heinzl and A. Ilderton, Opt. Commun. 282, 1879 (2009).
[30] A. Di Piazza, Lett. Math. Phys. 83, 305 (2008).

[31] C. Harvey, T. Heinzl, and M. Marklund, Phys. Rev. D 84, 116005 (2011).

[32] A. Ilderton and G. Torgrimsson, Phys. Rev. D 88, 025021 (2013).

[33] T. Heinzl, A. Ilderton, and M. Marklund, Phys. Lett. B 692, 250 (2010).

[34] F. Mackenroth, A. Di Piazza, and C. H. Keitel, Phys. Rev. Lett. 105, 063903 (2010).

[35] L. S. Brown and T. W. B. Kibble, Phys. Rev. 133, A705 (1964).

[36] A. Di Piazza, K. Z. Hatsagortsyan, and C. H. Keitel, Phys. Rev. Lett. 105, 220403 (2010).

[37] A. Noble, D. A. Burton, J. Gratus, and D. A. Jaroszynski, J. Math. Phys. 54, 043101 (2013). 